\title{
PLURISUBHARMONICALLY SEPARABLE COMPLEX MANIFOLDS
}

\author{
EVGENY A. POLETSKY AND NIKOLAY SHCHERBINA
}

\begin{abstract}
Let $M$ be a complex manifold and $P S H^{c b}(M)$ be the space of bounded continuous plurisubharmonic functions on $M$. In this paper we study when the functions from $P S H^{c b}(M)$ separate points. Our main results show that this property is equivalent to each of the following properties of $M$ :

(1) the core of $M$ is empty.

(2) for every $w_{0} \in M$ there is a continuous plurisubharmonic function $u$ with the logarithmic singularity at $w_{0}$.

Moreover, the core of $M$ is the disjoint union of the sets $E_{j}$ that are 1-pseudoconcave in the sense of Rothstein and have the following Liouville property: every function from $P S H^{c b}(M)$ is constant on each of $E_{j}$.
\end{abstract}

\section{IntRoduction}

Let $M$ be a complex manifold and $P S H^{c b}(M)$ be the space of bounded continuous plurisubharmonic functions on $M$. In this paper we study obstructions to separation of points in a complex manifold $M$ by functions from $P S H^{c b}(M)$.

There are complex manifolds, for example compact manifolds, where all plurisubharmonic functions are constants. There are parabolic manifolds, for example $\mathbb{C}^{n}$, where all bounded plurisubharmonic functions are constants. For their characterization see 9 and 1 . But there are plenty of complex manifolds like $\mathbb{D} \times \mathbb{C}, \mathbb{D}$ is the unit disk, where the space $P S H^{c b}(M)$ is large but, nevertheless, does not separate points.

The first main results of our paper can be summarized in the following theorem.

Theorem I. For a complex manifold $M$ the following statements are equivalent:

(1) the functions from $P S H^{c b}(M)$ separate points of $M$;

(2) for every point $w_{0} \in M$ there is a function $u \in P S H^{c b}(M)$ that is smooth and strictly plurisubharmonic near $w_{0}$;

(3) for every point $w_{0} \in M$ there are a negative continuous plurisubharmonic function $v$ on $M$ and constants $C_{1}$ and $C_{2}$ such that $\log \left|z-w_{0}\right|+C_{1}<$ $v(z)<\log \left|z-w_{0}\right|+C_{2}$ near $w_{0}$.

The main obstruction to separation is the $\operatorname{set} \mathbf{c}(M)$ of all points $w \in M$, where every function of $P S H^{c b}(M)$ fails to be smooth and strictly plurisubharmonic near $w$. It was first introduced and systematically studied by Harz-Shcherbina-Tomassini in [2]-4] and was called the core of $M$. Observe that directly from the definition one concludes that $\mathbf{c}(M)$ is a closed subset of $M$. Among the main properties of

2010 Mathematics Subject Classification. Primary 32U05; Secondary 32F10, 32U35.

Key words and phrases. plurisubharmonic functions, 1-pseudoconcave sets.

The first author was partially supported by a grant from Simons Foundation. 
the core established in these papers we mention here the following result that will be one of the important technical tools in the present paper.

Theorem. (see [2, Theorem 3.2]) Let $M$ be a complex manifold. Then the set $\mathbf{c}(M)$ is 1-pseudoconcave in the sense of Rothstein. In particular, $\mathbf{c}(M)$ is pseudoconcave in $M$ if $\operatorname{dim}_{\mathbb{C}} M=2$.

Our next main result is the following theorem that was proved in 3 when the dimension of $M$ is two.

Theorem II. Let $M$ be a complex manifold. Then the set $\mathbf{c}(M)$ is the disjoint union of the sets $E_{j}, j \in J$, that are 1-pseudoconcave in the sense of Rothstein and have the following Liouville property: every function from $P S H^{c b}(M)$ is constant on each of $E_{j}$.

The equivalence of statements 2 and 3 in Theorem I is proved in Theorem 3.2 The equivalence of 1 and 2 and Theorem II are proved in Theorem 4.8 Note that the sets $E_{j}$ can have a fractal nature (see [5]).

Observe that if $E \subset M$ is a set that is 1-pseudoconcave in the sense of Rothstein in $M$ which has the property that each bounded above continuous plurisubharmonic function $\varphi$ on $M$ is constant on $E$, then $E \subset \mathbf{c}(M)$ (for details see Lemma 3.1 in [3). Hence, mentioned above Theorem II clarify the phenomenon of both, existence and the structure of the core.

Note that in this paper we are dealing mainly with the core defined using continuous plurisubharmonic on $M$ functions (the core $\mathbf{c}^{0}(M)$ in terminology of [4]), while the main object of the study in 2-4 was the core $\mathbf{c}(M)$ defined using smooth plurisubharmonic on $M$ functions. Note also, that another proof of Theorem II for cores defined by smooth plurisubharmonic functions was obtained by Slodkowski [11 using essentially different methods. His proof also covers the case of minimal kernels which are defined and studied in 12 .

\section{BASIC FACTS}

Let $P S H(M)$ be the space of plurisubharmonic functions on a complex manifold $M$. We denote by $P S H^{b}(M)\left(P S H^{c}(M)\right)$ the subspaces of bounded above (continuous) functions in $P S H(M)$. Let $P S H^{c b}(M)$ be the intersection of $P S H^{b}(M)$ and $P S H^{c}(M)$.

The plurisubharmonic envelope $u$ of a function $\psi$ on a complex manifold $M$ is the supremum of all plurisubharmonic functions on $M$ that are less or equal to $\psi$ on $M$. If $\psi$ is upper semicontinuous, then by the Poletsky-Rosay theorem (see [7] and $[8]$ )

$$
u(z)=\inf _{f \in \mathcal{A}(\overline{\mathbb{D}}, M), f(0)=z} \frac{1}{2 \pi} \int_{0}^{2 \pi} \psi\left(f\left(e^{i \theta}\right)\right) d \theta .
$$

Given a complex manifold $M$, an open set $U \subset M$ and a point $w_{0} \in M$ let $v(z)$ be the supremum of all negative plurisubharmonic functions on $M$ that are less than -1 on $U$. The harmonic measure $\omega\left(w_{0}, U, M\right)$ of the set $U$ at $w_{0}$ is equal to $-v\left(w_{0}\right)$. By the Poletsky-Rosay theorem $\omega\left(w_{0}, U, M\right)$ is the supremum over all analytic disks $f: \overline{\mathbb{D}} \rightarrow M, f(0)=w_{0}$, of the normalized measure $\lambda$ of the set $\left\{\theta: 0 \leq \theta<2 \pi, f\left(e^{i \theta}\right) \in U\right\}$.

A closed set $E \subset M$ is called 1-pseudoconcave in the sense of Rothstein if for any $z_{0} \in E$ and for any strictly plurisubharmonic function $\rho$ defined on a neighborhood 
$V$ of $z_{0}$ at any neighborhood $U \subset \subset V$ containing $z_{0}$ there is a point $z \in E \cap U$ where $\rho(z)>\rho\left(z_{0}\right)$.

By their definition these sets are perfect, i.e., have no isolated points. But it may happen that such a set is compact. For example, take the unit ball $B$ in $\mathbb{C}^{2}$ and blow-up a complex projective line $E$ at the origin. We get a complex manifold $M$. Clearly, $E$ is a set that is 1-pseudoconcave in the sense of Rothstein.

But if $M$ is Stein, then any connected component $X$ of $E$ is non-compact. Indeed, by [3, Lemma 5.4], $X$ is a 1-pseudoconcave in the sense of Rothstein set. If $X$ is compact and $\rho$ is a smooth strictly plurisubharmonic exhaustion function on $M$, then we take the minimal $a$ such that $X \subset\{\rho(z) \leq a\}$. If $z_{0} \in X \cap\{\rho=a\}$, then $\rho(z) \leq \rho\left(z_{0}\right)$ for all $z \in X$ and it shows that $X$ is not a set that is 1-pseudoconcave in the sense of Rothstein.

\section{Cores And FUnCtions With LOGARIthmiC Singularities}

Let $M$ be a connected complex manifold and $w_{0} \in M$. Let us choose some local coordinates near $w_{0}$ and define the pluricomplex Green function with a pole at $w_{0}$ as

$g_{M}\left(z, w_{0}\right)=\sup \left\{u \in P S H(M): u<0\right.$ on $M, u(z)<\log \left|z-w_{0}\right|+c$ near $\left.w_{0}\right\}$.

The function $g_{M}$ is a plurisubharmonic in $z$ on $M$ and $g_{M}\left(w_{0}, w_{0}\right)=-\infty$.

Let $G_{M}\left(w_{0}, c\right)=\left\{z \in M: g\left(z, w_{0}\right)<c\right\}, c<0$, be Green balls. Evidently Green balls are open. They are also connected. Indeed, if the open set $G_{M}\left(w_{0}, c\right)$ has a connected component $U$ that does not contain $w_{0}$, then we take the function $u(z)$ equal to $c$ on $U$ and to $g_{M}\left(z, w_{0}\right)$ on $M \backslash U$. This function is, evidently, plurisubharmonic on $M \backslash \partial U$. But if $z \in \partial U$, then $g_{M}\left(z, w_{0}\right) \geq c$. Otherwise a neighborhood of $z$ will be in $U$. Hence $u$ is upper semicontinuous and, since $u \geq g_{M}$, plurisubharmonic. But $g_{M}\left(z, w_{0}\right) \geq u(z)$ on $M$ and, consequently, $g_{M}\left(z, w_{0}\right) \geq c$ on $U$. Hence $U$ does not lie in $G_{M}\left(w_{0}, c\right)$.

We will need the following lemma.

Lemma 3.1. Let $u$ be a bounded plurisubharmonic function on $M$ that is smooth and strictly plurisubharmonic near $w_{0} \in M$. Then for any coordinate neighborhood $V$ of $w_{0}$ there is a ball $U \subset V$ centered at $w_{0}$ and a pluriharmonic function $h$ on $U$ such that $u-h>0$ near $\partial U$ and $u\left(w_{0}\right)-h\left(w_{0}\right)<0$.

Proof. Let us choose some coordinate neighborhood $X \subset V$ around $w_{0}$ such that $u$ is smooth on $X$. For $z \in X$ we set

$$
L_{w_{0}}(z)=\sum_{i, j=1}^{n} u_{z_{i}, z_{j}}\left(w_{0}\right)\left(z_{i}-\left(w_{0}\right)_{i}\right)\left(z_{j}-\left(w_{0}\right)_{j}\right)
$$

and

$$
H_{w_{0}}(z)=\sum_{i, j=1}^{n} u_{z_{i}, \bar{z}_{j}}\left(w_{0}\right)\left(z_{i}-\left(w_{0}\right)_{i}\right)\left(\bar{z}_{j}-\left(\bar{w}_{0}\right)_{j}\right) .
$$

The Taylor expansion of $u$ at $w_{0}$ is

$$
u(z)=u\left(w_{0}\right)+2 \boldsymbol{R e}\left(\nabla u\left(w_{0}\right), z-w_{0}\right)+\boldsymbol{R e} L_{w_{0}}(z)+\frac{1}{2} H_{w_{0}}(z)+o\left(\left\|z-w_{0}\right\|^{2}\right) .
$$

The function

$$
v(z)=u\left(w_{0}\right)+2 \boldsymbol{R e}\left(\nabla u\left(w_{0}\right), z-w_{0}\right)+\boldsymbol{R e} L_{w_{0}}(z)
$$


is pluriharmonic on $X$ and there is a neighborhood $Y \subset \subset X$ such that the function $u(z)-v(z)>c\left|z-w_{0}\right|^{2}$ on $Y$ for some $c>0$. We take as $U$ a ball of radius $r$, centered at $w_{0}$ and compactly belonging to $Y$. Let $a=c r^{2} / 2$. If $h=v+a$, then the function $u-h>0$ on $\partial U$ and $u\left(w_{0}\right)-h\left(w_{0}\right)<0$.

Now we can prove the equivalence of statements 2 and 3 in Theorem I.

Theorem 3.2. A point $w_{0} \notin \mathbf{c}(M)$ if and only if there are a negative continuous plurisubharmonic function $v$ on $M$ and constants $C_{1}$ and $C_{2}$ such that $\log \left|z-w_{0}\right|+$ $C_{1}<v(z)<\log \left|z-w_{0}\right|+C_{2}$ near $w_{0}$.

Proof. Let $u$ be a continuous negative plurisubharmonic function on $M$ strictly plurisubharmonic near $w_{0}$. By Lemma 3.1 in some coordinate neighborhood $V$ of $w_{0}$ there is a ball $U$ of radius $r$ centered at $w_{0}$ and a pluriharmonic function $h$ on $V$ such that $u-h>0$ on $\partial U$ and $a=u\left(w_{0}\right)-h\left(w_{0}\right)<0$. There is another ball $B \subset \subset U$ of radius $s$ centered at $w_{0}$ such that $u-h<a / 2$ on $B$. The pluricomplex Green function $g_{U}\left(z, w_{0}\right)=\log \left(\left|z-w_{0}\right| / r\right)$ on $U$. We take a constant $c>0$ such that $c \log (s / r)>a / 2$. Then $c g_{U}>u-h$ on $\partial B$. Define the function $v$ on $M$ as $g_{U}+h / c$ on $B, \max \left\{g_{U}+h / c, u / c\right\}$ on $U \backslash B$ and $u / c$ outside of $U$. Since $g_{U}+h / c>u / c$ on $\partial B$ and $g_{U}+h / c<u / c$ on $\partial U$, the function $v$ is continuous and plurisubharmonic on $M$.

To prove the converse statement we take in some coordinate neighborhood of $w_{0}$ an open ball $B$ of radius $r$ centered at $w_{0}$ such that $\log \left|z-w_{0}\right|+C_{1}<v(z)<$ $\log \left|z-w_{0}\right|+C_{2}$ on $\bar{B}$. Let $B_{1}=B\left(w_{0}, r / 2\right)$. Then we take the function $\phi(z)=$ $a\left(\left|z-w_{0}\right|^{2}-r^{2}\right)$ on $B$, where $a>0$ is chosen so that $-3 a r^{2} / 4<\log (r / 2)+C_{1}$. This implies that $\phi<v$ on $\partial B_{1}$. We define the function $u$ on $M$ as $\max \{v, \phi\}$ on $B_{1}$ and as $v$ everywhere else. Clearly $u$ is a negative continuous plurisubharmonic function on $M$.

Since $v(z)<\log \left|z-w_{0}\right|+C_{2}$ near $w_{0}$, there is an open neighborhood $U$ of $w_{0}$ where $v<\phi$. Hence $u=\phi$ on $U$ and is smooth and strictly plurisubharmonic near $w_{0}$.

Corollary 3.3. If $w_{0} \notin \mathbf{c}(M)$, then $g_{M}\left(w, w_{0}\right)>\log \left|z-w_{0}\right|+C\left(w_{0}\right)$ in some coordinate neighborhood of $w_{0}$ for some constant $C\left(w_{0}\right)$.

The following lemma tells us that the notions of cores and maximal functions are related to each other.

Lemma 3.4. Let $\Omega \subset \subset M$ be a domain biholomorphic to a bounded domain in $\mathbb{C}^{n}$, $w_{0} \in \Omega$ and let $\phi \in P S H^{c b}(M)$. Let $u$ be a continuous plurisubharmonic function on $\Omega$ such that $\lim \sup _{z \rightarrow z_{0}, z_{0} \in \partial \Omega} u(z) \leq \phi\left(z_{0}\right)$. If $w_{0} \in \mathbf{c}(M)$, then $u\left(w_{0}\right) \leq \phi\left(w_{0}\right)$.

Proof. Suppose that $u\left(w_{0}\right)=\phi\left(w_{0}\right)+a, a>0$. Let $u_{1}=u-a / 2$ and let $U=\{z$ : $\left.u_{1}(z)>\phi(z)\right\}$. Then $U \subset \subset \Omega$. We take an open set $V \subset \subset \Omega$ such that $U \subset \subset V$ and $u_{1}<\phi-b$ on $\partial V$ for some $b>0$. Since $\Omega$ is biholomorphic to a domain in $\mathbb{C}^{n}$, there is a decreasing sequence of smooth plurisubharmonic functions $v_{j}$ on a neighborhood of $\bar{V}$ converging to $u_{1}$ on $\bar{V}$. If $j$ is sufficiently large, then we may assume that $v_{j}\left(w_{0}\right)>\phi\left(w_{0}\right)+a / 2$, but $v_{j}<\phi-b / 2$ on $\partial V$.

The domain $\Omega$ is biholomorphic to a bounded domain in $\mathbb{C}^{n}$. Hence there is a bounded, smooth and strictly plurisubharmonic function $v$ on $\Omega$. We set $u_{2}=$ $v_{j}+c v$, where $c>0$ is chosen in such a way that $u_{2}<\phi$ on $\partial V$ and $u_{2}\left(w_{0}\right)>\phi\left(w_{0}\right)$. We define the function $\psi$ as $\max \left\{u_{2}, \phi\right\}$ on $V$ and as $\phi$ on $M \backslash \bar{V}$. Since the function 
$\psi$ is continuous and plurisubharmonic on $M$, smooth and strictly plurisubharmonic near $w_{0}$, we see that $w_{0} \notin \mathbf{c}(M)$.

Corollary 3.5. If $\mathbf{c}(M)=M$, then any $\phi \in P S H^{c b}(M)$ is maximal.

\section{Foliation of CORES}

Let $w_{0}$ be a point in $M$. A point $z \in A^{b}\left(w_{0}\right)\left(A^{c b}\left(w_{0}\right)\right)$ if $u(z) \leq u\left(w_{0}\right)$ for any $u \in P S H^{b}(M)\left(u \in P S H^{c b}(M)\right)$. Evidently, $A^{b}\left(w_{0}\right) \subset A^{c b}\left(w_{0}\right)$.

Let us list some easily derived properties of the sets $A^{b}\left(w_{0}\right)$ and $A^{c b}\left(w_{0}\right)$.

Proposition 4.1. (1) The set $A^{c b}\left(w_{0}\right)$ is closed.

(2) If $z_{0} \in A^{b}\left(w_{0}\right)\left(z_{0} \in A^{c b}\left(w_{0}\right)\right)$, then $A^{b}\left(z_{0}\right) \subset A^{b}\left(w_{0}\right)\left(A^{c b}\left(z_{0}\right) \subset A^{c b}\left(w_{0}\right)\right)$.

(3) A point $w \in A^{b}\left(w_{0}\right)$ if and only if $\omega(w, U, M)=1$ for any neighborhood $U$ of $w_{0}$.

(4) If $w \in A^{b}\left(w_{0}\right)$, then $g_{M}\left(w, w_{0}\right)=-\infty$. Hence the set $A^{b}\left(w_{0}\right)$ is pluripolar if $g_{M}\left(\cdot, w_{0}\right) \not \equiv-\infty$.

(5) A point $w_{0} \notin \mathbf{c}(M)$ if and only if $A^{c b}\left(w_{0}\right)=\left\{w_{0}\right\}$.

(6) Any bounded holomorphic function on $M$ is constant on $A^{b}\left(w_{0}\right)$.

Proof. (1). If $u \in P S H^{c b}(M)$, then the set $\left\{w: u(w) \leq u\left(w_{0}\right)\right\}$ is closed. Since $A^{c b}\left(w_{0}\right)$ is the intersection of such sets over all $u \in P S H^{c b}(M),(1)$ follows.

(2) is evident. To show (3) we note that if $w \in A^{b}\left(w_{0}\right)$ and $U$ is a neighborhood of $w_{0}$, then any negative plurisubharmonic function on $M$ that is less or equal to -1 on $U$ is also less or equal to -1 at $w$. Hence $\omega(w, U, M)=1$.

If $\omega(w, U, M)=1$ for any neighborhood $U$ of $w_{0}$ and $u$ is a plurisubharmonic function on $M$ such that $u \leq A$ on $M$, then for any $\varepsilon>0$ we take a neighborhood $U$ of $w_{0}$, where $u \leq u\left(w_{0}\right)+\varepsilon$ on $U$. In view of the mentioned above Poletsky-Rosay theorem, it follows that there is an analytic disk $f$ such that $f(0)=w$ and the measure of the set $E=\left\{\theta: 0 \leq \theta<2 \pi, f\left(e^{i \theta}\right) \in U\right\}$ is greater than $1-\varepsilon$. Hence $u(w) \leq\left(u\left(w_{0}\right)+\varepsilon\right)(1-\varepsilon)+A \varepsilon$. Since $\varepsilon>0$ is arbitrary, we see that $u(w) \leq u\left(w_{0}\right)$.

Let us show (4). Since $g_{M}\left(w_{0}, w_{0}\right)=-\infty$, for any $N<0$ there is a neighborhood $U$ of $w_{0}$ such that $g_{M}\left(z, w_{0}\right) \leq N$ on $U$. If $w \in A^{b}\left(w_{0}\right)$, then $\omega(w, U, M)=1$ and for any $\varepsilon>0$ there is an analytic disk $f$ such that $f(0)=w$ and the measure of the set $E=\left\{\theta: 0 \leq \theta<2 \pi, f\left(e^{i \theta}\right) \in U\right\}$ is greater than $1-\varepsilon$. Hence $g_{M}\left(f(0), w_{0}\right) \leq$ $(1-\varepsilon) N$. Since $N$ and $\varepsilon$ are arbitrary, we see that $g_{M}\left(w, w_{0}\right)=-\infty$. Thus $g_{M}\left(w, w_{0}\right)=-\infty$ on $A^{b}\left(w_{0}\right)$ and if $g_{M}\left(\cdot, w_{0}\right) \not \equiv-\infty$, then $A^{b}\left(w_{0}\right)$ is pluripolar.

(5). If a point $w_{0} \notin \mathbf{c}(M)$, then by Theorem 3.2 there is a negative continuous function $u$ on $M$ such that $\{u=-\infty\}=\left\{w_{0}\right\}$. Hence $A^{c b}\left(w_{0}\right)=\left\{w_{0}\right\}$.

If $A^{c b}\left(w_{0}\right)=\left\{w_{0}\right\}$, then we take a ball $B$ centered at $w_{0}$ and for each point $z \in$ $\partial B$ find a function $u_{z} \in P S H^{c b}(M)$ such that $u_{z}\left(w_{0}\right)=0$ and $u_{z}(z)>0$. We take finitely many points $z_{1}, \ldots, z_{k}$ in $\partial B$ such that the function $u=\max \left\{u_{z_{1}}, \ldots, u_{z_{k}}\right\}$ is greater than 0 on $\partial B$. Then the maximal plurisubharmonic function $v$ on $B$ with boundary values equal to $u$ has the value at $w_{0}$ strictly bigger than $u\left(w_{0}\right)$. By Lemma $3.4 w_{0} \notin \mathbf{c}(M)$.

(6). Let $f$ be a bounded holomorphic function on $M$ and $|f|<r-1$ on $M$. If $w \in A^{b}\left(w_{0}\right)$, then $\log |f(w)+a| \leq \log \left|f\left(w_{0}\right)+a\right|$ for any $a \in \mathbb{C}$ with $|a|=r$. If $h(w)=(f(w)+a)^{-1}$, then again $\log |h(w)| \leq \log \left|h\left(w_{0}\right)\right|$. Hence $\left|f(w)+r e^{i \theta}\right|=$ $\left|f\left(w_{0}\right)+r e^{i \theta}\right|$ for any $\theta \in[0,2 \pi]$. Therefore

$$
|f(w)|^{2}-\left|f\left(w_{0}\right)\right|^{2}=2 \operatorname{Re}\left(\left(f\left(w_{0}\right)-f(w)\right) r e^{-i \theta}\right)
$$


and this implies that $f(w)=f\left(w_{0}\right)$.

In Theorem 4.3 below we prove the major property of the sets $A^{c b}$. For its proof we need the following lemma.

Lemma 4.2. Suppose that $A$ is a closed set in the closure $\bar{B}$ of the unit ball $B \subset \mathbb{C}^{n}$ centered at the origin, $0 \in A$, and $\rho$ is a strictly plurisubharmonic function defined on an open neighborhood of $\bar{B}$ such that $\rho(0)=0$ and $\rho \leq 0$ on $A$. Then there are $z_{1} \in A \cap B$ and a strictly plurisubharmonic function $\rho_{1}$ defined on an open neighborhood $U$ of $z_{1}$ such that $\rho_{1}\left(z_{1}\right)=0, \rho_{1} \leq 0$ on $A \cap U$ and $\nabla \rho_{1}\left(z_{1}\right) \neq 0$.

Proof. Since the function $\rho$ is strictly plurisubharmonic, there is $\varepsilon>0$ such that the function $\rho^{*}(z)=\rho(z)-\varepsilon\|z\|^{2}$ is also strictly plurisubharmonic on $\bar{B}$. Observe that the function $\rho^{*} \leq 0$ on $A, \rho^{*}(0)=0$ and, moreover, now we also have that $\rho^{*} \leq-\varepsilon<0$ on $A \cap \partial B$. If $\rho^{*} \leq 0$ on some open ball centered at the origin, then it is zero on this ball and this is impossible since $\rho^{*}$ is strictly plurisubharmonic. Hence we can take a point $a$ with $\rho^{*}(a)>0$ so close to the origin that for all $t \in[0,1]$ we have $\rho *<-\varepsilon / 2<0$ on $(A+t a) \cap \partial B$. Consider now the function $F:[0,1] \rightarrow[0, \infty)$ defined as

$$
F(t)=\max \left\{\rho^{*}(z): z \in(A+t a) \cap \bar{B}\right\} .
$$

It is obvious that the function $F$ is continuous and $\left[0, \rho^{*}(a)\right] \subset F([0,1])$. By Sard's theorem there is a non-critical value $s \in\left[0, \rho^{*}(a)\right]$ for the function $\rho^{*}$. Let $t^{*} \in[0,1]$ be the value of the parameter $t$ such that $F\left(t^{*}\right)=s$ and let $z^{*}$ be a point in $A+t^{*} a$, where $\rho^{*}$ attains its maximum equal to $s$. Since $\rho^{*}<0$ on $(A+t a) \cap \partial B, z^{*} \in B$, and since $\rho^{*}\left(z^{*}\right)>0$ the point $z_{1}=z^{*}-t^{*} a$ is also in $B$. Hence $z_{1}$ and the function $\rho_{1}(z)=\rho^{*}\left(z+t^{*} a\right)-s$, satisfy all requirements of the lemma.

Now we can prove the theorem.

Theorem 4.3. If $A^{c b}\left(w_{0}\right) \neq\left\{w_{0}\right\}$, then the set $A^{c b}\left(w_{0}\right)$ is 1-pseudoconcave in the sense of Rothstein.

Proof. Suppose to get a contradiction that there are $z_{0} \in A^{c b}\left(w_{0}\right)$, a neighborhood $U \subset \subset M$ of $z_{0}$ and a strictly plurisubharmonic function $\rho$ defined on an open neighborhood of $\bar{U}$ such that the set $A^{c b}\left(w_{0}\right) \cap \bar{U} \cap\left\{\rho>\rho\left(z_{0}\right)\right\}$ is empty. By Lemma 4.2 we may assume that in some local coordinates $\left(z_{1}, \ldots, z_{n}\right)$ the set $U$ is a ball $B(0,1)$ in $\mathbb{C}^{n}, z_{0}=0, \rho(0)=0$ and $\nabla \rho(0) \neq 0$. Then there is a biholomorphic change of coordinates near $z_{0}$ such that the function $\rho$ becomes strictly convex and $z_{0}$ is still equal to 0 . We may assume that $\nabla \rho(0)=(1,0, \ldots, 0)$.

First, we consider the case when $z_{0} \neq w_{0}$ and $w_{0} \notin B$. Let $h(z)=\operatorname{Re} z_{1}$ and $S_{0}=\{\rho=0\}$. There is a smaller ball $B=B(0, r)$ and $\delta>0$ such that $\rho\left(z+t e_{1}\right)>0$ when $0<t \leq \delta$ and $z \in S_{0} \cap B(0, r)$. The Taylor expansion of $\rho$ at $z_{0}$ is

$$
\rho(z)=2 h(z)+Q(z)+o\left(\|z\|^{2}\right),
$$

where $Q(z) \geq a\|z\|^{2}, a>0$, is a real quadratic form. If $\rho(z)<0$, then $a\|z\|^{2} \leq$ $-2 h(z)+o\left(\|z\|^{2}\right)$ and there is $\varepsilon>0$ such that the set $G=\{z \in B:-\varepsilon<$ $h(z)<0, \rho(z)<0\}$ compactly belongs to $B$. We take $0<\delta_{1}<\min \{\delta, \varepsilon / 2\}$ so small that the set $G_{1}=G+\left(\delta_{1}, 0, \ldots, 0\right)$ also compactly belongs to $B$. Let $S=\partial G_{1} \cap\left\{S_{0}+\delta_{1} e_{1}\right\}$.

Observe that $\rho>0$ on $S$ and, therefore, $S \cap A^{c b}\left(w_{0}\right)=\emptyset$. So for every $z \in S$ there is a function $u_{z} \in P S H^{c b}(M)$ such that $u_{z}\left(w_{0}\right)=0$ and $u_{z}(z)>0$. Replacing 
$u_{z}$ with $\max \left\{u_{z}, 0\right\}$ we may assume that $u_{z} \geq 0$ on $M$. By compactness of $S$, there are points $z_{1}, \ldots, z_{k}$ on $S$ such that the function $u=\max \left\{u_{z_{1}}, \ldots, u_{z_{k}}\right\}>\alpha>0$ on $S$.

Recall that $h=\delta_{1}-\varepsilon<0$ on $\partial G_{1} \backslash S$. If $\delta_{2}=\delta_{1}-\varepsilon$ and $m$ is the infimum of $u$ on $S$, then the function

$$
v(z)=\frac{m}{2 \varepsilon}\left(h(z)-\delta_{2}\right)
$$

is pluriharmonic on $\bar{G}_{1}, v=0$ on $\partial G_{1} \backslash S, v>0$ on $G_{1}$ and

$$
v(z) \leq v\left(\delta_{1}, 0, \ldots, 0\right)=m / 2
$$

on $S$. Thus $v \leq u$ on $\partial G_{1}$.

We define the function $u_{1}$ on $M$ as $\max \{u, v\}$ on $\bar{G}_{1}$ and $u$ on $M \backslash \bar{G}_{1}$. This function will be plurisubharmonic on $M$ and $u_{1}\left(z_{0}\right)>0$. Hence $z_{0} \notin A^{c b}\left(w_{0}\right)$ and we came to a contradiction. Therefore, the set $A^{c b}\left(w_{0}\right)$ is 1-pseudoconcave in the sense of Rothstein at any point $z_{0} \in A^{c b}\left(w_{0}\right), z_{0} \neq w_{0}$.

If $z_{0}=w_{0}$ and there are a neighborhood $U$ of $z_{0}$ and a strictly plurisubharmonic function $\rho$ defined on $U$ and such that the set $A^{c b}\left(w_{0}\right) \cap U \cap\left\{\rho>\rho\left(w_{0}\right)\right\}$ is empty, then we perform the same construction and note that $G_{1}$ is biholomorphic to a bounded domain in $\mathbb{C}^{n}$ containing $w_{0}$ and a plurisubharmonic function $v$ on $\bar{G}_{1}$ has its boundary values less or equal to $u$ while $v\left(w_{0}\right)>u\left(w_{0}\right)$. Hence by Lemma 3.4 $w_{0} \notin \mathbf{c}(M)$ and by $(5) A^{c b}\left(w_{0}\right)=\left\{w_{0}\right\}$.

Let $A_{e}^{c b}\left(w_{0}\right)$ be the set of $w \in M$ such that $u(w)=u\left(w_{0}\right)$ for any $u \in P S H^{c b}(M)$.

Lemma 4.4. If $w_{0} \in \mathbf{c}(M)$, then there is a function $u \in P S H^{c b}(M)$ equal to 0 on $A_{e}^{c b}\left(w_{0}\right)$ and negative on $A^{c b}\left(w_{0}\right) \backslash A_{e}^{c b}\left(w_{0}\right)$.

Proof. For any point $w \in A^{c b}\left(w_{0}\right) \backslash A_{e}^{c b}\left(w_{0}\right)$ there is a function $u_{w} \in P S H^{c b}(M)$ such that $u_{w}\left(w_{0}\right)=0$ and $u_{w}(w)<0$. Replacing $u_{w}$ with $\max \left\{u_{w},-1\right\}$ we may assume that the uniform norm $\left\|u_{w}\right\|$ of each function $u_{w}$ is finite.

Let us take a countable family of points $w_{k} \in A^{b}\left(w_{0}\right) \backslash A_{e}^{c b}\left(w_{0}\right)$ and their neighborhoods $U_{k}$ such that $\cup U_{k}=A^{c b}\left(w_{0}\right) \backslash A_{e}^{c b}\left(w_{0}\right)$ and $u_{w_{k}}<0$ on $U_{k}$. Note that $u_{w_{k}} \leq 0$ on $A^{c b}\left(w_{0}\right)$ and $u_{w_{k}}=0$ on $A_{e}^{c b}\left(w_{0}\right)$.

Let $b_{k}=\left\|u_{w_{k}}\right\|$. We find $\gamma_{k}>0$ such that the series $\sum_{k=1}^{\infty} \gamma_{k} b_{k}$ converges. Let $u=\sum_{k=1}^{\infty} \gamma_{k} u_{w_{k}}$. Then $u \in P S H^{c b}(M), u=0$ on $A_{e}^{c b}\left(w_{0}\right)$ and $u<0$ on $A^{c b}\left(w_{0}\right) \backslash A_{e}^{c b}\left(w_{0}\right)$.

The following lemma is the analog of [10, Lemma 2.2] that can be used on complex manifolds and is proved as in the original.

Lemma 4.5. Let $K$ be a compact set in a complex manifold $M$. Suppose that there is a bounded smooth strictly plurisubharmonic function $\rho$ defined on a neighborhood $V$ of $K$. Let $u$ be a plurisubharmonic function on $V$. Assume that there is a nonempty compact set $L \subset K$ such that $\max _{K} u>\max _{L} u$. Then there are a point $w^{*}$ in $K \backslash L$, a neighborhood $U$ of $w^{*}$ and a smooth strictly plurisubharmonic function $v$ on $U$ such that $v\left(w^{*}\right)=0$ and $v(w)<0$ when $w \neq w^{*}$ belongs to $K \cap U$.

Proof. There is $\varepsilon>0$ such that $M=\max _{K} u_{\varepsilon}>\max _{L} u_{\varepsilon}$ for the function $u_{\varepsilon}=$ $u+2 \varepsilon \rho$. Take $w_{1} \in K$ such that $u_{\varepsilon}\left(w_{1}\right)=M$. Then $w_{1} \in K \backslash L$.

We can use the same argument as in Lemma 3.1 and find a sufficiently small coordinate ball $U$ centered at $w_{1}$ and a pluriharmonic function $h$ on $U$ such that the function $\rho_{1}=\rho+h$ attains its strict minimum at $w_{1}$ and $\rho_{1}\left(w_{1}\right)=0$. Let 
$v_{0}=u_{\varepsilon}-\varepsilon \rho_{1}-M$. Then $v_{0}\left(w_{1}\right)=0, v_{0}(w)<0$ when $w \neq w_{1}$ belongs to $K \cap U$, and $v_{0}<a<0$ on $\partial U$. Let us take a decreasing sequence of smooth plurisubharmonic functions $u_{j}, j \in \mathbb{N}$, converging to $u$ on an open neighborhood of $\bar{U}$. Then the strictly plurisubharmonic functions $v_{j}=u_{j}+\varepsilon(\rho+h)-M$ decreasingly converge to $v_{0}$. Hence there is $j$ such that $v_{j}<a / 2$ on $\partial U$ while $v_{j}\left(w_{1}\right) \geq 0$. We take $w^{*} \in K \cap U$, where $v_{j}$ attains its maximum $M^{\prime}$ on $K \cap \bar{U}$ and let $v(w)=$ $v_{j}(w)-M^{\prime}-\delta\left\|w-w^{*}\right\|^{2}$, where $\delta>0$ is a sufficiently small number.

Following the terminology of [10] we say that a closed set $X$ in a complex manifold $M$ has the local maximum property if $X$ is perfect (i.e. has no isolated points) and for any $z_{0} \in X$ there is an open neighborhood $V$ of $z_{0}$ in $M$ with compact closure such that if an open set $U \subset \subset V$ contains $z_{0}$ and the set $L=X \cap \partial U$ is non-empty, then

$$
\sup _{X \cap \bar{U}} u \leq \sup _{L} u
$$

for any plurisubharmonic function $u$ on $V$.

Theorem 4.6. If $M$ is a complex manifold, then any closed set $X \subset M$ has the local maximum property if and only if it is 1-pseudoconcave in the sense of Rothstein.

Proof. Let $X$ be a set that is 1-pseudoconcave in the sense of Rothstein and $w_{0} \in X$. Take an open coordinate neighborhood $V$ of $w_{0}$. Suppose that $U \subset \subset V$ is an open set containing $w_{0}$ and the set $L=X \cap \partial U$ is non-empty. If $u$ is a plurisubharmonic function on $V$ such that $\sup _{X \cap \bar{U}} u>\sup _{L} u$, then by Lemma4.5 there are a point $w^{*}$ in $X \cap U$, a neighborhood $W$ of $w^{*}$ and a strictly plurisubharmonic function $v$ on $W$ such that $v\left(w^{*}\right)=0$ and $v(w)<0$ when $w \neq w^{*}$ belongs to $X \cap W$. But this contradicts the assumption that the set $X$ is a 1-pseudoconcave in the sense of Rothstein. Hence $X$ has the local maximum property.

Conversely, if $X$ has the local maximum property and is not 1-pseudoconcave in the sense of Rothstein, then there is a point $w_{0} \in X$ and a strictly plurisubharmonic function $\rho$ defined on a neighborhood $V$ of $w_{0}$ such that $\rho(w) \leq \rho\left(w_{0}\right)$ when $w \in$ $X \cap V$. We may assume that $V$ lies in a coordinate ball centered at $w_{0}$ and find $\varepsilon>0$ such the function $u(w)=\rho(w)-\varepsilon\left|w-w_{0}\right|^{2}$ is still strictly plurisubharmonic. Since $X$ is perfect there is an open set $U \subset \subset V$ that contains $w_{0}$ such that the set $L=X \cap \partial U$ is non-empty. Hence, $\sup _{L} u<u\left(z_{0}\right)$ and this contradiction shows that $X$ is 1-pseudoconcave in the sense of Rothstein.

Theorem 4.7. If $M$ is a complex manifold and $w_{0} \in \mathbf{c}(M)$, then the set $A_{e}^{c b}\left(w_{0}\right)$ has the local maximum property.

Proof. It is clear that the set $A_{e}^{c b}\left(w_{0}\right)$ is closed. Since $A_{e}^{c b}\left(w_{0}\right)=A_{e}^{c b}\left(w_{1}\right)$ for any $w_{1} \in A_{e}^{c b}\left(w_{0}\right)$, it suffice to prove the theorem at $w_{0}$. Let $U$ be an open set with compact closure containing $w_{0}$ and the set $L=A^{c b}\left(w_{0}\right) \cap \partial U$ is non-empty. Let us show that the set $A_{e}^{c b}\left(w_{0}\right) \cap \partial U$ is also non-empty.

Since, in view of Theorem 4.3 the set $A^{c b}\left(w_{0}\right)$ is 1-pseudoconcave in the sense of Rothstein, it has the local maximum property. By Lemma 4.4 there is a continuous function $u$ on $M$ equal to 0 on $A_{e}^{c b}\left(w_{0}\right)$ and negative on $A^{c b}\left(w_{0}\right) \backslash A_{e}^{c b}\left(w_{0}\right)$. If the set $A_{e}^{c b}\left(w_{0}\right) \cap \partial U$ is empty, then $\sup _{A^{c b}\left(w_{0}\right) \cap \bar{U}} u>\sup _{L} u$ and this contradicts to the local maximum property of $A^{c b}\left(w_{0}\right)$. Hence $A_{e}^{c b}\left(w_{0}\right) \cap \partial U \neq \emptyset$ and since the set $A^{c b}\left(w_{0}\right)$ is perfect, the set $A_{e}^{c b}\left(w_{0}\right)$ is also perfect. 
Now let $V \subset M$ be an open set with compact closure, an open set $U \subset \subset V$ contains $w_{0}$ and the set $A_{e}^{c b}\left(w_{0}\right) \cap \partial U$ is non-empty. Suppose that there is a plurisubharmonic function $v$ defined on $V$ such that $\sup _{A_{e}^{c b}\left(w_{0}\right) \cap \bar{U}} v>\sup _{A_{e}^{c b}\left(w_{0}\right) \cap \partial U} v$. We may assume that $\sup _{A_{e}^{c b}\left(w_{0}\right) \cap \bar{V}} v=0$ so the function $v$ is negative on $A_{e}^{c b}\left(w_{0}\right) \cap \partial U$. There is a neighborhood $W$ of the latter set such that $v$ is negative on this neighborhood. Let $u$ be a function from Lemma 4.4. The set $K=\left(A^{c b}\left(w_{0}\right) \cap \partial U\right) \backslash W$ is compact and does not contain points of $A_{e}^{c b}\left(w_{0}\right)$ and, therefore, there is a constant $a>0$ such that the function $v_{1}=v+a u$ is negative on this set. Since $v$ is negative on $W$ and $u$ is non-positive on $A^{c b}\left(w_{0}\right)$, the function $v_{1} \leq-\varepsilon$ on $A^{c b}\left(w_{0}\right) \cap \partial U$ for some $\varepsilon>0$. Also $\sup _{A_{e}^{c b}\left(w_{0}\right) \cap \bar{U}} v_{1}=0$ because $u=0$ on $A_{e}^{c b}\left(w_{0}\right)$. Hence $\sup _{A^{c b}\left(w_{0}\right) \cap \bar{U}} v_{1}>\sup _{A^{c b}\left(w_{0}\right) \cap \partial U} v_{1}$. But the set $A^{c b}\left(w_{0}\right)$ has the local maximum property and this means that we got a contradiction. Therefore, the set $A_{e}^{c b}\left(w_{0}\right)$ has the local maximum property.

As a corollary to the latter result we obtain the following theorem.

Theorem 4.8. Let $M$ be a complex manifold with non-empty core $\mathbf{c}(M)$. Then:

(1) for every $w_{0} \in M$ the set $A_{e}^{c b}\left(w_{0}\right) \neq\left\{w_{0}\right\}$ if and only if $w_{0} \in \mathbf{c}(M)$;

(2) if $w_{0} \in \mathbf{c}(M)$, then the set $A_{e}^{c b}\left(w_{0}\right)$ is a is 1-pseudoconcave in the sense of Rothstein, lies in $\mathbf{c}(M)$ and all functions $u \in P S H^{c b}(M)$ are constants on $A_{e}^{c b}\left(w_{0}\right)$;

(3) the core $\mathbf{c}(M)$ of $M$ can be decomposed into the disjoint union of closed sets $E_{j}, j \in J$, that are 1-pseudoconcave in the sense of Rothstein and have the following Liouville property: Every function $\varphi \in P S H^{c b}(M)$ is constant on each of the sets $E_{j}$.

Proof. (1) If $w_{0} \in \mathbf{c}(M)$, then by Theorem 4.7 the set $A_{e}^{c b}\left(w_{0}\right)$ has the local maximum property and, therefore, is not equal to $\left\{w_{0}\right\}$. Conversely, if $w_{0} \notin \mathbf{c}(M)$, then $A^{c b}\left(w_{0}\right)=\left\{w_{0}\right\}$ by Proposition $4.1(5)$ and, consequently, $A_{e}^{c b}\left(w_{0}\right)=\left\{w_{0}\right\}$.

(2) By Theorems 4.6 and 4.7 the set $A_{e}^{c b}\left(w_{0}\right)$ is 1-pseudoconcave in the sense of Rothstein. Let $w_{1} \in A_{e}^{c b}\left(w_{0}\right)$ and $w_{1} \neq w_{0}$. Then $w_{0} \in A_{e}^{c b}\left(w_{1}\right)$. Hence $A_{e}^{c b}\left(w_{1}\right) \neq\left\{w_{1}\right\}$ and $w_{1} \in \mathbf{c}(M)$. By the definition of the sets $A_{e}^{c b}(M)$ all functions $u \in P S H^{c b}(M)$ are constants on $A_{e}^{c b}\left(w_{0}\right)$.

(3) If $A_{e}^{c b}\left(w_{0}\right) \cap A_{e}^{c b}\left(w_{1}\right) \neq \emptyset$, then $A_{e}^{c b}\left(w_{0}\right)=A_{e}^{c b}\left(w_{1}\right)$. Hence the relation $w_{0} \sim w_{1}$ if $w_{1} \in A_{e}^{c b}\left(w_{0}\right)$ is an equivalence relation. Let $J=\mathbf{c}(M) / \sim$ and $[w]_{\sim}$ be the equivalence class of $w \in \mathbf{c}(M)$. Then $\mathbf{c}(M)$ is the disjoint union of the sets $E_{j}=A_{e}^{c b}(w),[w]_{\sim}=j$.

If the manifold $M$ is not Stein, it may happen that the sets $A^{c b}\left(w_{0}\right)$ and $A_{e}^{c b}\left(w_{0}\right)$ are compact. For example, take the unit ball $B$ in $\mathbb{C}^{2}$ and blow-up a complex projective line $X$ at the origin. We get a complex manifold $M$ and a holomorphic mapping $F$ of $M$ onto $B$ such that $F(X)=\{0\}$. If $u$ is a plurisubharmonic function on $B$, then the function $v=u \circ F$ is plurisubharmonic on $M$ while any plurisubharmonic function on $M$ is constant on $X$. Hence $\mathbf{c}(M)=X$ and $A^{c b}\left(w_{0}\right)=$ $A_{e}^{c b}\left(w_{0}\right)=X$ for $w_{0} \in X$.

The following theorem shows that such phenomenon can exist only on non-Stein manifolds. 
Theorem 4.9. Let $M$ be a complex manifold and let $X \neq\left\{w_{0}\right\}$ be the closed connected component of $A^{c b}\left(w_{0}\right)$ containing $w_{0}$. If $X$ is compact, then $A^{c b}\left(w_{0}\right)=X$ and $M$ is not Stein.

Proof. If $X$ is compact, we can take a decreasing sequence of open neighbourhoods $V_{p}$ of $X$ with compact closure such that $\bigcap_{p=1}^{\infty} V_{p}=X$ and $\partial V_{p} \cap A^{c b}\left(w_{0}\right)=\emptyset$ for every $p \in \mathbb{N}$ (for the existence of the sets $V_{p}$ see, for example, Proposition 2 on page 108 of [NN]). If we fix a set $V_{p}$ from this sequence, then, since points $w \in \partial V_{p}$ do not belong to $A^{c b}\left(w_{0}\right)$, for each of them there is a function $u_{w} \in P S H^{c b}(M)$ such that $u_{w}(w)>0$ and $u_{w}\left(w_{0}\right)=0$. Note that $u_{w} \leq 0$ on $A^{c b}\left(w_{0}\right)$. Let us pick up finitely many points $w_{k}, 1 \leq k \leq m$, on $\partial V_{p}$ such that the function $u=\max \left\{u_{w_{1}}, \ldots, u_{w_{m}}\right\}$ is positive on $\partial V_{p}$. It follows from the compactness of $\partial V_{p}$ that $u>\alpha$ on $\partial V_{p}$ for some $\alpha>0$.

Let us take the function $v$ on $M$ that is equal to $u$ on $V_{p}$ and to $\max \{u, \alpha / 2\}$ on $M \backslash V_{p}$. This function will be plurisubharmonic and $v(w)>v\left(w_{0}\right)=0$ for $w \in M \backslash V_{p}$. Hence $A^{c b}\left(w_{0}\right) \subset V_{p}$. Then finally from $\bigcap_{p=1}^{\infty} V_{p}=X$ we conclude that $A^{c b}\left(w_{0}\right)=X$.

\section{EXAmPle}

Here we present an example which refutes the following possible conjectures about the sets $\mathbf{c}(M), A^{b}\left(w_{0}\right)$ and $A^{c b}\left(w_{0}\right)$ :

(1) the set $A^{b}\left(w_{0}\right)$ is closed;

(2) the set $A^{b}\left(w_{0}\right)=A^{c b}\left(w_{0}\right)$;

(3) if $w_{1} \in A^{b}\left(w_{0}\right)$, then $w_{0} \in A^{b}\left(w_{1}\right)$;

(4) if $w_{0} \in \mathbf{c}(M)$, then there is a set $E$ containing $w_{0}$ and at least one more point such that any $u \in P S H^{b}(M)$ is constant on $E$.

Let

$$
\phi(\zeta)=\sum_{k=1}^{\infty} \gamma_{k} \log \left|\left(\zeta-k^{-1}\right) / 2\right|,
$$

where all $\gamma_{k}>0$ are chosen so that the series converges for all $\zeta \in \mathbb{C}, \zeta \neq k^{-1}$, $k \in \mathbb{N}, \phi(0)=-3$ and $\phi(\zeta)<-2$ on the unit disk $\mathbb{D}$. Clearly the function $\phi$ is subharmonic. Consider the domain

$$
\Omega=\left\{\left(z_{1}, z_{2}\right) \in \mathbb{C}^{2}: \psi\left(z_{1}, z_{2}\right)<0\right\},
$$

where

$$
\psi\left(z_{1}, z_{2}\right)=\left|z_{1}\right|^{2}+\left|z_{2}\right|^{2}+\log \left|\left(z_{1}-1 / 2\right) / 2\right|+\phi\left(z_{2}\right) .
$$

This domain contains the bidisk $\mathbb{D}^{2}$ and the lines $L_{k}=\left\{z_{2}=k^{-1}\right\}, k \in \mathbb{N}$, and the line $L_{0}=\left\{z_{1}=1 / 2\right\}$. If $u$ is a bounded above plurisubharmonic function on $\Omega$, then $u$ is constant on each of these lines. Hence by connectedness, it is constant on the union $X$ of this lines.

Moreover, if $X_{0}=\left\{\left(z_{1}, 0\right) \in \Omega\right\}$, i.e., if $\left|z_{1}\right|^{2}+\log \left|\left(z_{1}-1 / 2\right) / 2\right|<3$, then $e^{\psi}$ is continuous and bounded on $\Omega$ and it is smooth and strictly plurisubharmonic outside of $X_{0} \cup X$. This shows that $\mathbf{c}(\Omega) \subset X_{0} \cup X$. On the other hand, clearly, $X \subset \mathbf{c}(\Omega)$ and, since cores are closed, it follows that $X_{0} \cup X \subset \mathbf{c}(\Omega)$.

To refute the conjecture (4) we take $w_{0}=(0,0)$. If $w \notin \mathbf{c}(\Omega)$, then, by Corollary 3.3. the function $g_{M}(w, w)=-\infty$ while $g_{M}\left(w_{0}, w\right)>-\infty$. If $w \in X$, then $\psi(w)=$ 
$-\infty$ while $\psi\left(w_{0}\right)=-3-\log 4$. If $w \in X_{0}$, then the family

$$
\psi_{a, b}\left(z_{1}, z_{2}\right)=a\left|z_{1}-b\right|^{2}+\left|z_{2}\right|^{2}+\log \left|\left(z_{1}-1 / 2\right) / 2\right|+\phi\left(z_{2}\right)
$$

for properly chosen $0<a<1$ and $b \in \mathbb{C}$ will consist of the bounded above on $\Omega$ plurisubharmonic functions that separate the points of $X_{0}$ from $w_{0}$.

If $u \in P S H^{b}(\Omega)$, then for any $\varepsilon>0$ there is a neighborhood $U$ of $(0,0)$ such that $u\left(z_{1}, z_{2}\right)<u(0,0)+\varepsilon$ on $U$. Since $U$ meets some lines $z_{2}=k^{-1}$, we see that $u<u(0,0)+\varepsilon$ on $X$. Thus $u \leq u(0,0)$ on $X$ by arbitrariness of $\varepsilon>0$ and, thereofore, $X \subset A^{b}((0,0))$. However, for the function $\psi$ we have $\psi<0$ on $\Omega$, $\psi(0,0)=-3-\log 4$ and $\psi\left(z_{1}, k^{-1}\right)=-\infty$. Thus $(0,0) \notin A^{b}(w)$ for any $w \in X$ and this refutes $(3)$.

If $w_{0} \in X$, then $A^{b}\left(w_{0}\right)=X$ because $\psi=-\infty$ holds only on $X$. The closure of $X$ contains $(0,0)$ and this means that $A^{b}\left(w_{0}\right)$ is not closed and is a proper subset of $A^{b}((0,0))$ while $(0,0) \in A^{c b}\left(w_{0}\right)$. This refutes (1) and (2).

\section{OPEN PROBLEMS}

(1) Is $A^{c b}\left(w_{0}\right)=A_{e}^{c b}\left(w_{0}\right)$ ?

(2) Does $A^{c b}\left(w_{0}\right) \subset \mathbf{c}(M)$ ?

(3) Is there a non-negative $u \in P S H^{c b}(M)$ such that the set $\{u=0\}=\mathbf{c}(M)$ ?

\section{REFERENCES}

[1] A. Aytuna and A. Sadullaev, Parabolic Stein manifolds, Math. Scand. 114 (2014), 86-109.

[2] T. Harz, N. Shcherbina, and G. Tomassini, On defining functions and cores for unbounded domains I, Math. Z. 286 (2017), 987-1002.

[3] T. Harz, N. Shcherbina, and G. Tomassini, On defining functions and cores for unbounded domains II, J. Geom. Anal. (2017), First Online: 21 April 2017.

[4] T. Harz, N. Shcherbina, and G. Tomassini, On defining functions and cores for unbounded domains III, submitted for publication.

[5] T. Harz, N. Shcherbina, and G. Tomassini, Wermer type sets and extension of CR functions, Indiana Univ. Math. J. 61 (2012), 431-459.

[6] R. Narasimhan and Y. Nievergelt, Complex analysis in one variable, 2nd edition. Birkhäuser, Boston, 2001.

[7] E.A. Poletsky, Plurisubharmonic functions as solutions of variational problems, Several complex variables and complex geometry, Part 1 (Santa Cruz, CA, 1989), 163-171, Proc. Sympos. Pure Math., 52, Part 1, Amer. Math. Soc., Providence, RI, 1991.

[8] J.P. Rosay, Poletsky theory of disks on holomorphic manifolds, Indiana Univ. Math. J. 52 (2003), 157-170.

[9] J.-P. Rosay, Discs in complex manifolds with no bounded plurisubharmonic functions, Proc. Amer. Math. Soc. 132 (2004), 2315-2319.

[10] Z. Slodkowski, Local maximum property and q-plurisubharmonic functions in uniform algebras, J. Math. Anal. Appl. 115 (1986), 105-130.

[11] Z. Slodkowski, Preliminary notes, Spring 2017.

[12] Z. Slodkowski and G. Tomassini, Minimal kernels of weakly complete spaces, J. Funct. Anal. 210 (2004), 125-147.

Department of Mathematics, Syracuse University, 215 Carnegie Hall, Syracuse, Ny 13244, USA

E-mail address: eapolets@syr.edu

Department of Mathematics, University of Wuppertal, 42119 Wuppertal, Germany

E-mail address: shcherbina@math.uni-wuppertal.de 